\title{
He-Ne Laser Imaging Probes Premature Diabetes Mellitus
}

\author{
Alok Singh ${ }^{1}$, Savita Singh ${ }^{1}$, Pradeep Kumar Singh ${ }^{2}$, N. S. Parmar ${ }^{3}$, Neetu Singh ${ }^{4}$ \\ ${ }^{1}$ Department of Physics, PSIT College of Engineering, Kanpur, Uttar Pradesh, India \\ ${ }^{2}$ Department of Mathematics, PSIT College of Engineering, Kanpur, Uttar Pradesh, India \\ ${ }^{3}$ Department of Mechanical Engineering, PSIT College of Engineering, Kanpu, Uttar Pradesh, India \\ ${ }^{4}$ Department of Chemistry, PSIT College of Engineering, Kanpur, Uttar Pradesh, India
}

Email address:

dr.singhalok@gmail.com (A. Singh)

\section{To cite this article:}

Alok Singh, Savita Singh, Pradeep Kumar Singh, N. S. Parmar, Neetu Singh. He-Ne Laser Imaging Probes Premature Diabetes Mellitus. International Journal of Biomedical Materials Research. Vol. 3, No. 2, 2015, pp. 14-18. doi: 10.11648/j.ijbmr.20150302.11

\begin{abstract}
An empirical approach to develop a comprehensive monitoring framework to track progress of ballistic photons through glucose doped aqueous humor by two dimensional sixteen Mueller polarization matrix element images for deepening efforts in polarimetry reported. The glucose doped aqueous humor has been illuminated with a He-Ne (632.8 nm) laser in vitro measurement. The obtained transmitted Mueller polarization matrix images are strong evidence to discriminate normal glucose level, impaired fasting glycaemia (IFG), impaired glucose tolerance (IGT) and diabetes mellitus.
\end{abstract}

Keywords: Polarimeter, Mueller Polarization Matrix, Aqueous Humor

\section{Introduction}

Interest in frequent determination of glucose concentrations of diabetic patients has increased in a compressed time frame since the publication of the diabetes control and complications trial report. Use of non invasive techniques offers boundless advantages, such as the absence of pain and exposure to sharp objects and biohazard materials, the potential for increased frequency of testing, and hence, tighter control of the glucose concentrations, and the potential for a closed loop system including a monitor and insulin pump[1,2]. Several publications and reviews have discussed the importance of non invasive glucose testing and report on attempts at its measurement [3-8]. The polarimetric measurements in turbid media have gained importance by their ability to provide information through photons. For instance, polarization imaging has been used to enhance spatial resolution in turbid samples and to increase contrast for surface imaging by removing the depolarization intensity that consists of multiply scattered photons [9].

A noninvasive body glucose monitoring device is defined as a device that comes in contact with, or remotely senses, a human body part, without protrusion through membranes or sampling a body fluid for analysis external to the body part. A noninvasive glucose monitor processes optical signals transmitted through or reflected by the stratum cornea, dermis and epidermis layers, subcutaneous tissue, interstitial fluid, and blood vessels which represents independent compartments. An empirical approach involves the collection of non-invasive signals from non diabetic individuals and diabetic patients while performing an oral glucose tolerance test (OGTT) or a glucose challenge test, simultaneously measure blood glucose concentrations by an invasive method and compute models based on the correlation between measured glucose values and noninvasive optical signals [10, 11].

Finally, the detection system and the measurement method are applied to body parts. The in vivo signals are again correlated with the invasive data by use of chemometric techniques. This stepwise approach allows for identification of noise components so that strategies may be derived to minimize their contribution to the signal before the use of chemometric techniques [12].

In this paper, we analyzed the polarization states of ballistic photons for characterization of aqueous humor at different glucose level. The aqueous humor is a transparent fluid similar to plasma located in the anterior and posterior chambers of the eye, the space between lens and the cornea. The ballistic photons preserve their polarization after passing through the aqueous humor sample. Thus, Mueller matrix polarization component of transmitted photons provides useful information about the glucose doped aqueous humor medium. 


\section{Theory}

An arbitrary polarization of light beam may be represented by a stokes vector $(\mathrm{S})$ as,

$$
S=\left[\begin{array}{l}
I \\
Q \\
U \\
V
\end{array}\right]
$$

where, I represents the total light intensity, Q and U represent the linearly polarized components of the beam, and $\mathrm{V}$ represents the circularly polarized component. For an arbitrary light beam, these components are related by,

$$
I^{2} \geq Q^{2}+U^{2}+V^{2}
$$

where, the equality and inequality hold for fully polarized light and partially polarized light respectively. The total degree of polarization $\left(\mathrm{DP}_{\mathrm{T}}\right)$ is given as,

$$
D P_{T}=\sqrt{\frac{Q^{2}+U^{2}+V^{2}}{I^{2}}}
$$

The linearly polarized fraction is represented by the degree of linear polarization $\left(\mathrm{DP}_{\mathrm{L}}\right)$ as,

$$
D P_{L}=\sqrt{\frac{Q^{2}+U^{2}}{I^{2}}}
$$

The degree of circularly polarization $\left(\mathrm{DP}_{\mathrm{C}}\right)$ is similarly defined as,

$$
D P_{C}=\sqrt{\frac{V^{2}}{I^{2}}}
$$

Light interaction with any optical element or medium is described as a multiplication of stokes vector with a $4 \times 4$ matrix, $S^{\prime}=$ MS. This sixteen element matrix is called Mueller matrix given as,

$$
[M]=\left(\begin{array}{llll}
m_{11} & m_{12} & m_{13} & m_{14} \\
m_{21} & m_{22} & m_{23} & m_{24} \\
m_{31} & m_{32} & m_{33} & m_{34} \\
m_{41} & m_{42} & m_{43} & m_{44}
\end{array}\right)
$$

The forty nine intensity measurements with various orientations of polarizer, analyzer and retarder are used to obtain sixteen elements of the Mueller matrix as shown in table 1 .

Table 1. Calculation of the sixteen Mueller matrix image orientations.

\begin{tabular}{llll}
\hline$[\mathrm{M} 11]$ & {$[\mathrm{M} 12]$} & {$[\mathrm{M} 13]$} & {$[\mathrm{M} 14]$} \\
$\mathrm{HH}+\mathrm{HV}+\mathrm{VH}+\mathrm{VV}$ & $\mathrm{HH}+\mathrm{HV}-\mathrm{VH}-\mathrm{VV}$ & $\mathrm{PH}+\mathrm{PV}-\mathrm{MH}-\mathrm{MV}$ & RH+RV-LH-LV \\
{$[\mathrm{M} 21]$} & {$[\mathrm{M} 22]$} & {$[\mathrm{M} 23]$} & {$[\mathrm{M} 24]$} \\
$\mathrm{HH}-\mathrm{HV}+\mathrm{VH}-\mathrm{VV}$ & $\mathrm{HH}-\mathrm{HV}-\mathrm{VH}+\mathrm{VV}$ & $\mathrm{PH}-\mathrm{PV}-\mathrm{MH}-\mathrm{MV}$ & $\mathrm{RH}-\mathrm{RV}-\mathrm{LH}+\mathrm{LV}$ \\
{$[\mathrm{M} 31]$} & {$[\mathrm{M} 32]$} & {$[\mathrm{M} 33]$} & {$[\mathrm{M} 34]$} \\
$\mathrm{HP}-\mathrm{HM}+\mathrm{VP}-\mathrm{VM}$ & $\mathrm{HP}-\mathrm{HM}-\mathrm{VP}+\mathrm{VM}$ & {$[\mathrm{M} 43]$} & $\mathrm{RP}-\mathrm{RM}-\mathrm{LP}+\mathrm{LM}$ \\
{$[\mathrm{M} 41]$} & {$[\mathrm{M} 42]$} & PR-PL-MR+ML & {$[\mathrm{M} 44]$} \\
HR-HL+VR-VL & HR-HL-VR+VL & RR-RL-LR+LL \\
\hline
\end{tabular}

\section{Experimental Procedure}

The polarimetric measurements were performed with the experimental setup as shown schematically in fig.1. The unpolarised He-Ne laser beam of $25 \mathrm{~mW}$ of wavelength 632.8 $\mathrm{nm}$ was passed through optical elements with aqueous humor. The optical elements consisted of two branches, input optical elements $\left(\mathrm{L}_{1}, \mathrm{P}_{1}\right.$, and $\left.\mathrm{W}_{1}\right)$ and output optical elements $\left(\mathrm{W}_{2}, \mathrm{P}_{2}\right.$, and $\mathrm{L}_{2}$ ), these optical elements were necessary to generate the input and output polarization states required to derive the sixteen output Mueller matrix of transmitted intensity.

The calibration of optical elements presented in the fig. 1 was done by a nulling technique involving the use of the input polarizer $\left(\mathrm{P}_{1}\right)$ and output polarizer $\left(\mathrm{P}_{2}\right)$ oriented with their principal axes at $90^{\circ}$ with respect to each other. Each component was calibrated by adjusting the supplied intensity until the desired input and output polarization states of $\mathrm{H}$ (Horizontal), V (Vertical), P $\left(+45^{0}\right), \mathrm{M}\left(-45^{0}\right)$ was obtained by the detection of a minimum intensity by the CCD camera. The calibration was done sequentially with the output retardation plate $\left(\mathrm{W}_{2}\right)$. To obtain the desired intensities the necessary degree of rotation from the output rotator for each of the desired polarization states, was determined by varying the state of the input polarizer $\left(\mathrm{P}_{1}\right)$ until the desired intensity was captured by the CCD camera. A similar calibration was done for retardation plate input polarization $\left(\mathrm{W}_{1}\right)$.

The laser beam was focused through lens $L_{1}(f=15 \mathrm{~cm})$ to the polarizer $P_{1}$ to generate linearly polarized light. The linearly polarized light from $\mathrm{P}_{1}$ was focused to retardation plate $\mathrm{W}_{1}(\lambda / 4$ plate $)$ behind the $\mathrm{P}_{1}$ to generate circularly polarized light, with the retarder principal plane at $45^{\circ}$ with respect to the plane of the incident linearly polarized beam. Light transmitted from aqueous humor was then pass through retarder $\mathrm{W}_{2}$ and analyzer $\mathrm{P}_{2}$ and focused by lens $\mathrm{L}_{2}$ on the CCD camera.

The intensity was detected by a charge coupled device CCD camera (Pulnix TM-6X-CCIR, Imager 1/2" interline transfer CCD, pixel - 752x582, scanning - 625 lines CCR, TV resolution $-560 \times 420$ ). The obtained images on CCD camera 
were analyzed via a MATLAB ${ }^{\circledR}$ program which performs calculation according to table 1 to generate the sixteen Mueller matrix element images.

Sample

In vitro experiment, the sample was an aqueous humor filled in $10 \mathrm{~mm}$ across rectangular glass cuvette. The composition of aqueous humor is indicated in Table 2. The aqueous humor was analyzed with three different concentration of glucose level given below.

Table 2. Composition of aqueous humor.

\begin{tabular}{ll}
\hline Item & Quantity \\
\hline Protein & $100 \mathrm{mmol} / 1$ \\
Sodium & $95 \mathrm{mmol} / 1$ \\
Lactic Acid & $65 \mathrm{mmol} / 1$ \\
Ascorbic Acid & $70 \mathrm{mmol} / 1$ \\
\hline
\end{tabular}

The aqueous humor is analyzed with three different concentration of glucose level given below.

a) For normal glucose level (4.5 $\mathrm{mmol} / \mathrm{l})$, aqueous humor was prepared with $81 \mathrm{mg}$ of glucose per deciliter of water and the corresponding Mueller matrix images shown in fig. 2.

b) For impaired fasting glycaemia $(6.1 \mathrm{mmol} / \mathrm{l})$, aqueous humor was prepared with $110 \mathrm{mg}$ of glucose per deciliter of water and the corresponding Mueller matrix images shown in fig. 3.

c) For impaired glucose tolerance $(7.0 \mathrm{mmol} / \mathrm{l})$, aqueous humor was prepared with $128 \mathrm{mg}$ of glucose per deciliter of water and the corresponding Mueller matrix images shown in fig. 4.

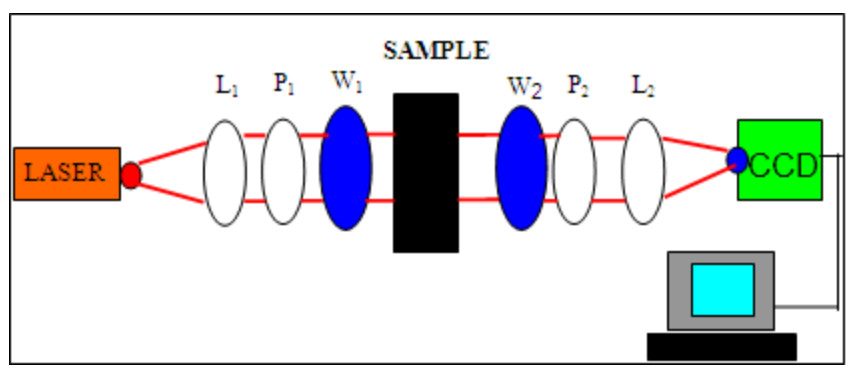

Figure 1. Experimental setup to measure the polarization states of transmitted light by Mueller matrix image elements.

\section{Results and Discussion}

A total of ten experiments with each glucose doped aqueous humor sample were conducted repeatedly to obtain accurate experimental results. The two dimensional Mueller matrix images of the glucose concentration taken for aqueous humor solution by polarimetric setup are shown in Fig 1, Fig 2 and Fig 3.

The $M_{11}$ element of the matrix shows the direct intensity pattern and the characteristic of the illuminating light. The other matrix is the results of linear and circular polarization output. These images and the matrix were acquired through forty nine orientations, and the measurements of the polarized optics are given in table 1 . Mueller matrices $\mathrm{M}_{22}, \mathrm{M}_{33}$ and $\mathrm{M}_{44}$ are unitary and represent the horizontal, vertical, \pm 45 , L-circular and R-circular polarization.

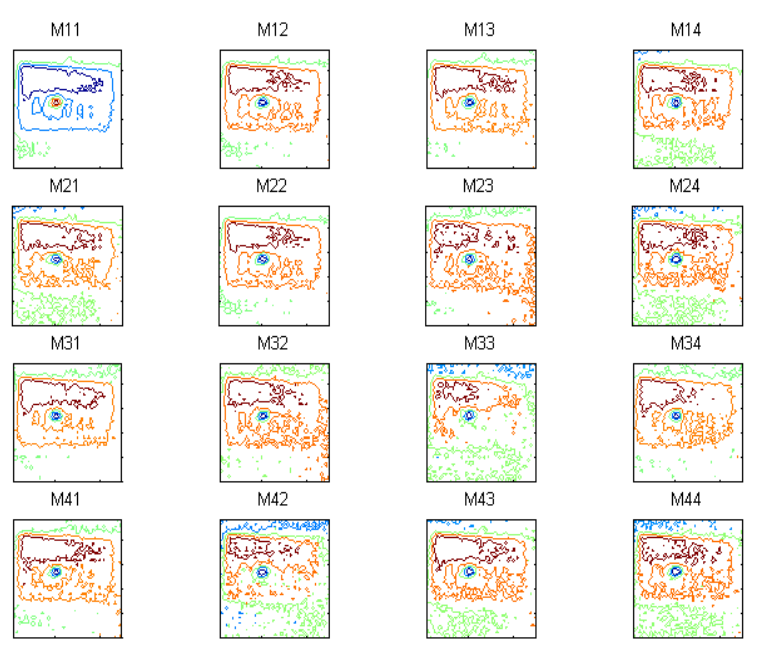

Figure 2. The two dimensional images of Mueller matrix elements for aqueous humor at $4.5 \mathrm{mmol} / \mathrm{l}$ glucose concentration.

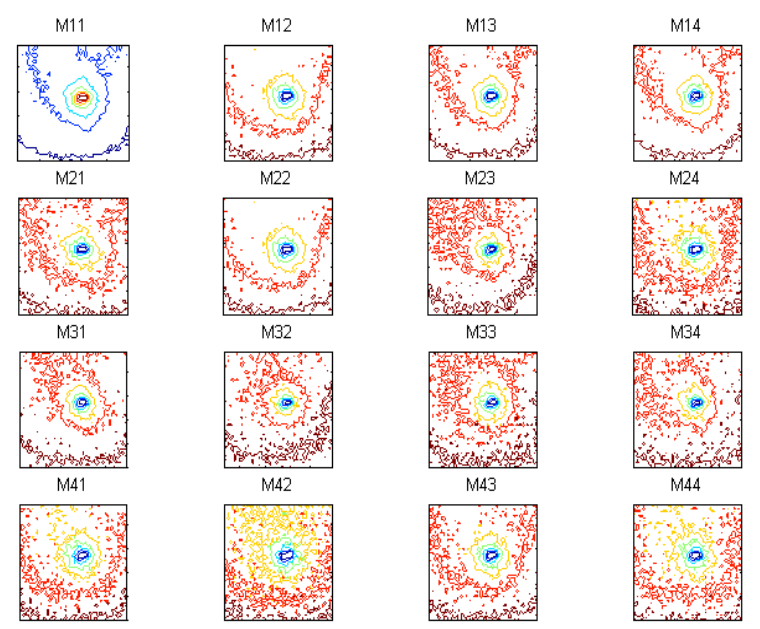

Figure 3. The two dimensional images of Mueller matrix elements for aqueous humor at $6.1 \mathrm{mmol} / \mathrm{l}$ glucose concentration.
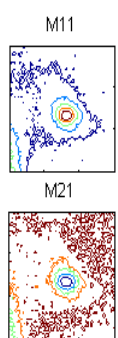

M31

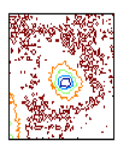

M41

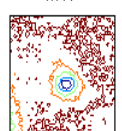

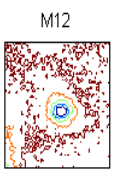
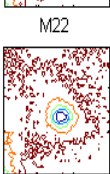

M32

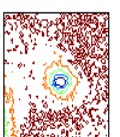

M42

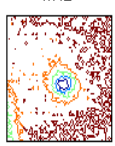

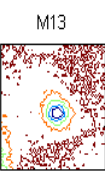

M23

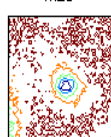

M33

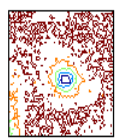

M43

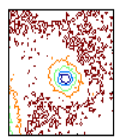

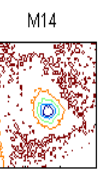

M24

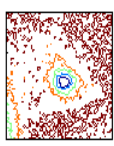

M34

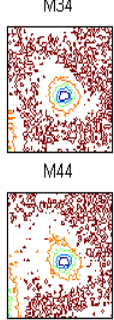

Figure 4. The two dimensional images of Mueller matrix elements for aqueous humor at $7.0 \mathrm{mmol} / \mathrm{l}$ glucose concentration. 
By observing at diagonal matrix image variations of the Mueller train and significant change in Mueller matrix images of $\mathrm{M}_{23}$ and $\mathrm{M}_{41}$ with increasing concentrations of glucose one can estimate the glucose concentration in aqueous humor. Similarly, continuous variation of $\mathrm{M}_{24}$ and diagonal Mueller matrix images in fig.2, fig.3, and fig.4 can be used to differentiate the glucose concentration in aqueous humor. Thus, a comparative study of optical images at different glucose levels (fig.2, fig.3, and fig.4) describes that as the glucose concentration in aqueous humor increases, the intensity of diagonal matrix of Mueller train at different glucose level decreases.

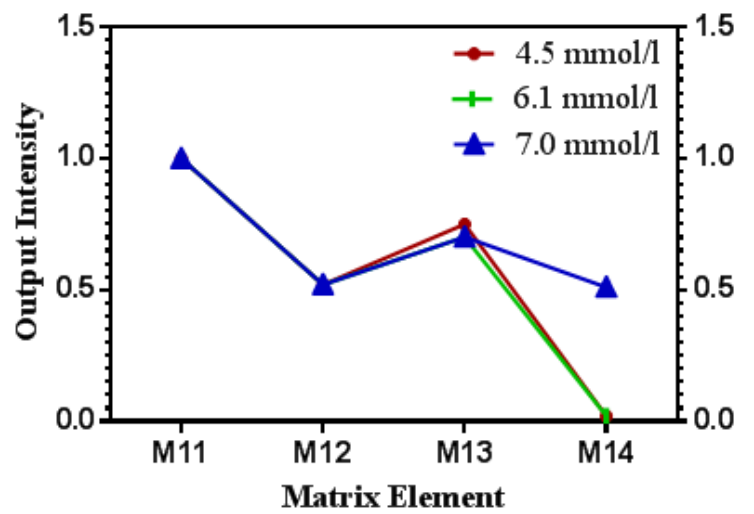

Figure 5. Output intensity versus Mueller matrix first row element curve.

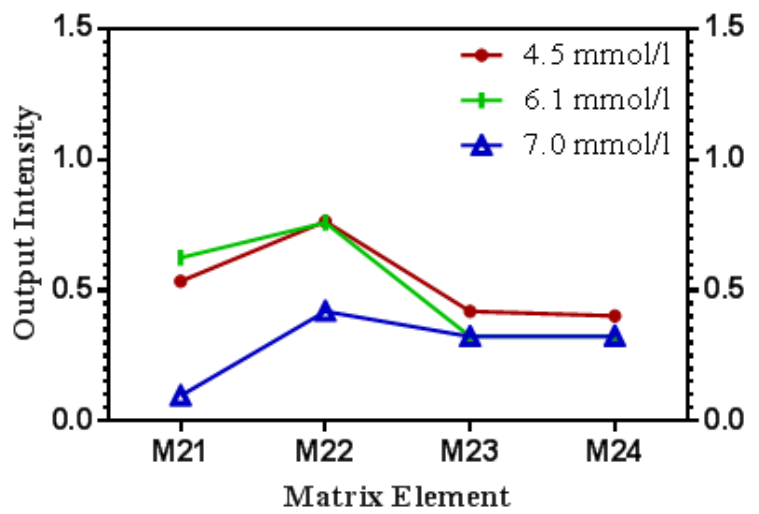

Figure 6. Output intensity versus Mueller matrix second row element curve.

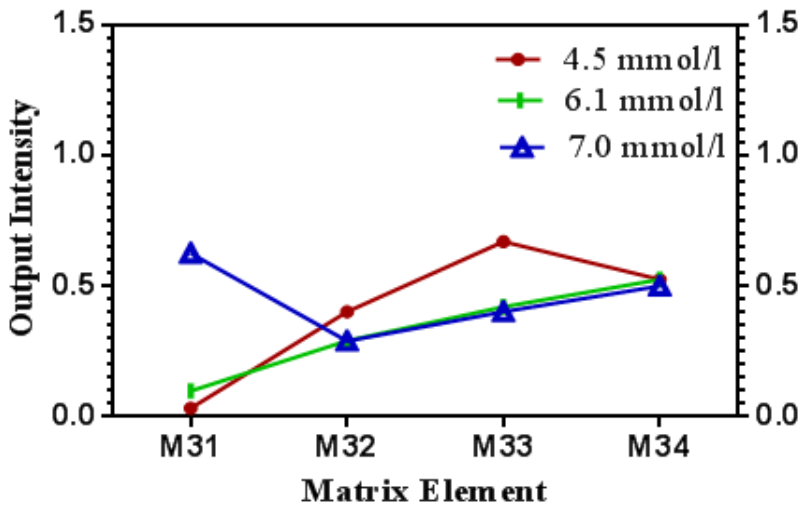

Figure 7. Output intensity versus Mueller matrix third row element curve.

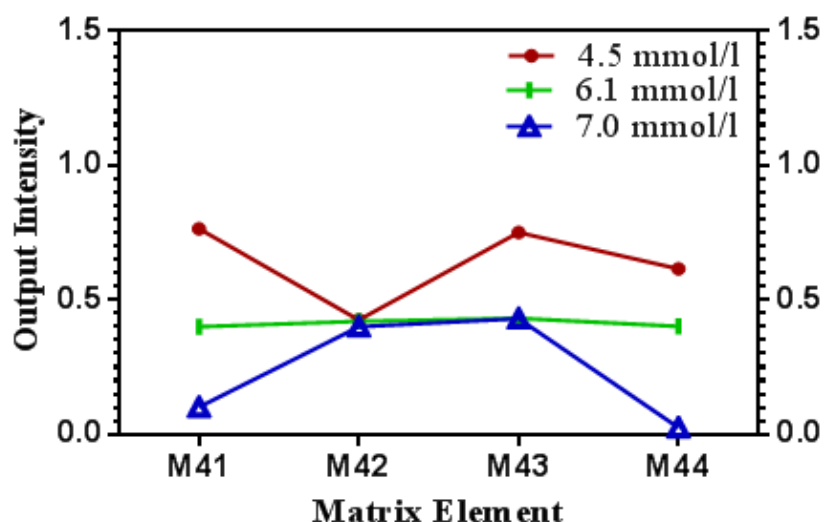

Figure 8. Output intensity versus Mueller matrix fourth row element curve.

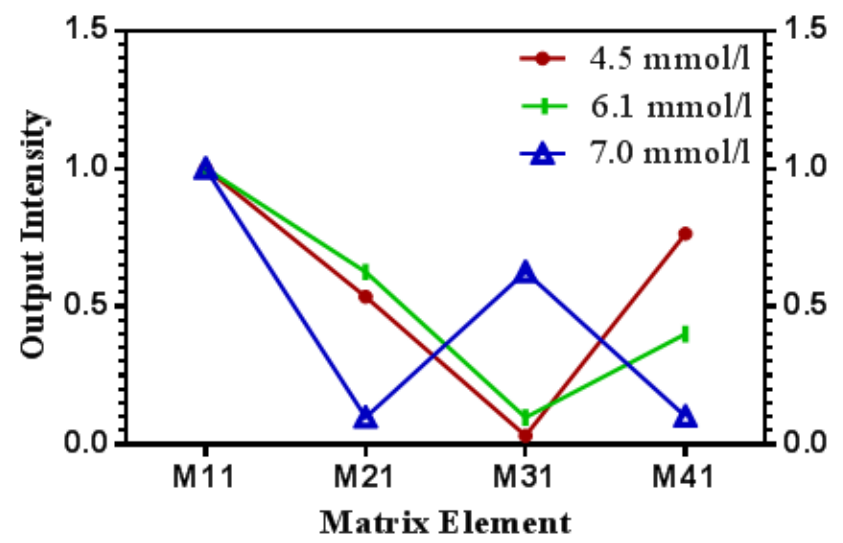

Figure 9. Output intensity versus Mueller matrix first column element curve.

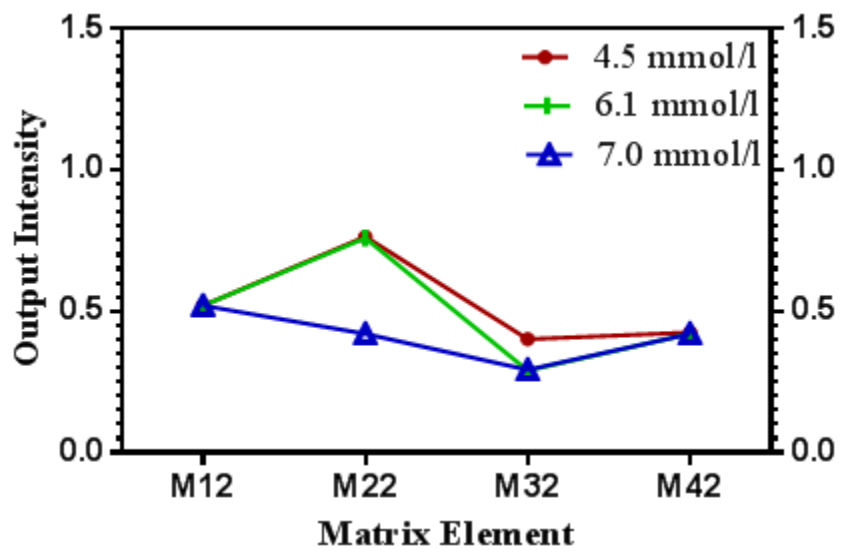

Figure 10. Output intensity versus Mueller matrix second column element curve.

Finally, the above qualitative image-data analysis facilitates a special stimulus for plotting an intrinsic pattern of obtained output intensity versus Mueller matrix row-wise element curves as shown in fig.5, fig.6, fig.7, and fig.8, while the intrinsic pattern of obtained output intensity versus Mueller matrix column-wise element curves as shown in fig.9, fig.10, fig.11, and fig.12. Output intensity versus Mueller matrix diagonal element curve is shown in fig. 13 . 


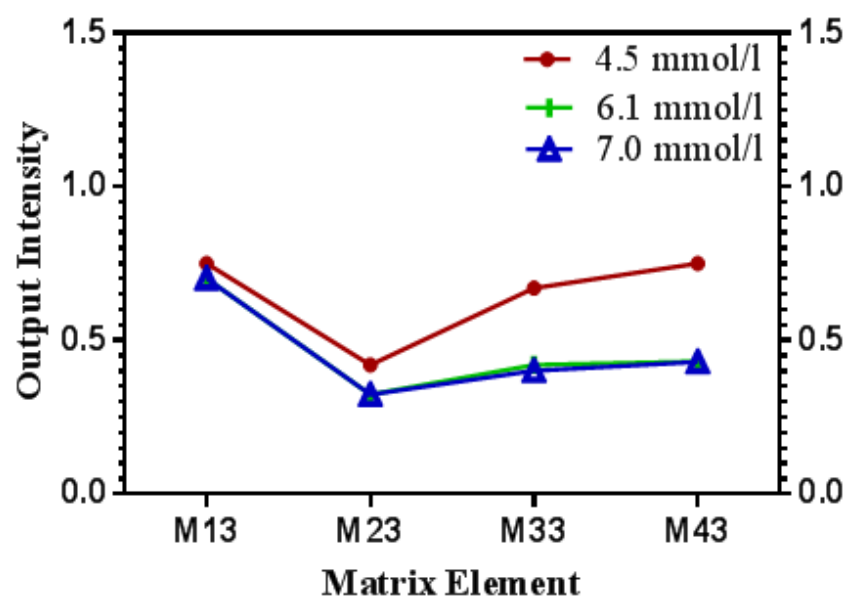

Figure 11. Output intensity versus Mueller matrix third column element curve.

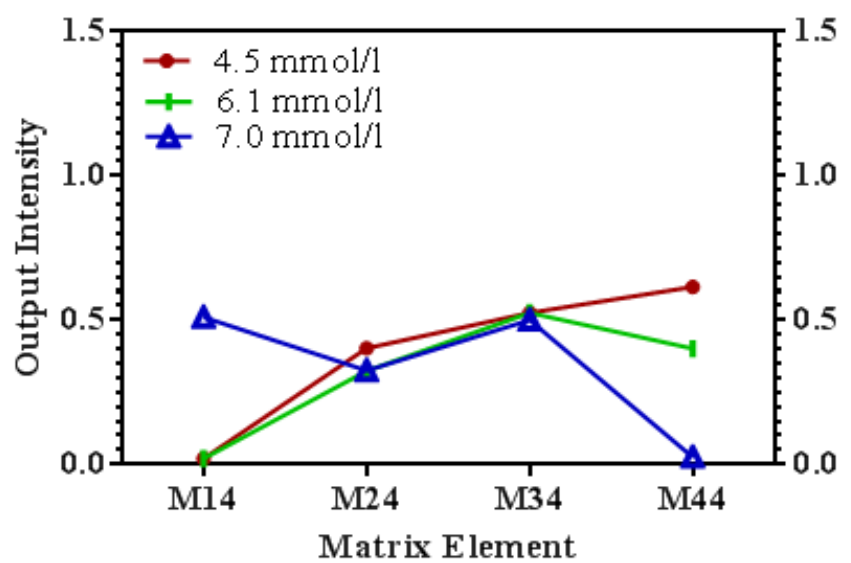

Figure 12. Output intensity versus Mueller matrix fourth column element curve.

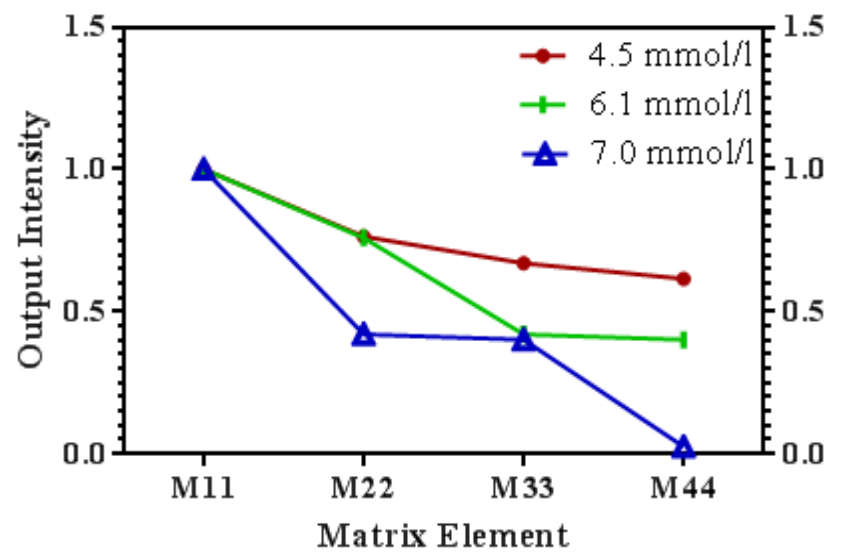

Figure 13. Output intensity versus Mueller matrix diagonal element curve.

The above curves display the ballistic photons through aqueous humor preserve the linear polarization state (See Fig 5, Fig 6 and Fig 7) while circular polarization state depolarizes with an increase of glucose concentration in aqueous humor (See Fig 8, and Fig 9). Thus, the preferential retention of linear polarization state over circular by ballistic photon through glucose doped aqueous humor has been illustrated by above curves. An easy discrimination among normal glucose level
(4.5 mmol/l), impaired fasting glycaemia (6.1 $\mathrm{mmol} / \mathrm{l})$, impaired glucose tolerance $(7.0 \mathrm{mmol} / \mathrm{l})$ can be made by observing the Fig 9 and Fig 13.The other complexity such as, fasting plasma glucose below $6.1 \mathrm{mmol} / 1$. , fasting levels between 6.1 and $7.0 \mathrm{mmol} / \mathrm{l}$ are borderline of impaired fasting glycaemia (IFG), and fasting levels repeatedly at or above 7.0 $\mathrm{mmol} / \mathrm{l}$ with diabetes can be determined by analyzing Fig 8 and Fig 12 .

\section{Conclusions}

The experimental characterization of glucose doped aqueous humor leads to the following conclusions,

1. The two dimensional transmitted Mueller matrix element images can estimate the glucose concentration in aqueous humor.

2. These images are capable to diagnose premature diabetes mellitus.

3. The output intensity versus Mueller matrix element curves and Mueller matrix element images distinguish normal glucose level, impaired fasting glycaemia (IFG), impaired glucose tolerance (IGT) and diabetes mellitus.

4. The output intensity versus Mueller matrix element curves are capable to give information about fasting plasma glucose level, impaired fasting glycaemia and fasting levels at or above with diabetes.

5. The output intensity versus Mueller matrix element curves illustrates the preferential retention of linear polarization state over circular polarization by ballistic photons through glucose doped aqueous humor.

6. This monitoring framework can also be used to determine optical properties of various biological phantoms using software programs.

Consequently, after nano-scale miniaturization of this non invasive glucose sensor can be located at the other side of cornea for detecting the optical rotation of the radiation that passed through the cornea to monitor glucose concentrations in diabetic patients regularly.

\section{Acknowledgements}

We are gratefully acknowledged to Optics and Photonics Research Laboratory (OPRL), PSIT College of Engineering, Kanpur, INDIA for providing optical images and testing facility.

\section{References}

[1] The Diabetes control and complications trial research group. "The effect of intensive treatment of diabetes on the development and progression of long-term complication in insulin-dependent diabetes mellitus." N Engl J Med.; 329(14): 977-86 (1993).

[2] The diabetes control and complications trial research group. "Life time benefits and costs of intensive therapy as practiced in the diabetes control and complications trial." JAMA; 276(17):1409-15 (1996). 
[3] DC. Klonoff, "Non-invasive blood glucose monitoring" Diabetes Care; 20(3):433-7 (1997).

[4] HM Heise, "Non-invasive monitoring of metabolites using near infrared spectroscopy: state of the art." Horm Metab Res.; 28(10):527-34 (1996).

[5] Andrea Tura, Alberto Maran, Giovanni Pacini ,"Non-invasive glucose monitoring: Assessment of technologies and devices according to quantitative criteria." Diabetes Research and Clinical Practice; 77(1):16-40 (2007)

[6] MR Robinson, RP Eaton, DM Haaland et al, "Nonivasive glucose monitoring in diabetic patients: a preliminary evaluation." Clinical Chemistry; 38(9): 1618-1622 (1992).

[7] McNichols, Roger J., and Gerard L. Cote, "Optical glucose sensing in biological fluids: an overview." Journal of Biomedical Optics; 5(1): 5-16 (2000).
[8] Goldstein, Dennis H, "Mueller matrix dual-rotating retarder polarimeter." Applied Optics; 31(31):6676-6683, (1992).

[9] J.M. Schmitt, A.H. Gandjbakhche, and R.F. Bonner, "Use of polarized light to discriminate short-path photons in a multiply scattering medium." Applied Optics; 31(30): 6535-6546(1992).

[10] Lide, R. David "“CRC handbook of physics and chemistry." The Chemical Rubber Company, Cleveland, OH (2003).

[11] Azzam, R. M. A., N. M. Bashara, and Stanley S. Ballard," Ellipsometry and Polarized Light." North Holland, (1978).

[12] Jacques, Steven L, "Monte Carlo modeling of light transport in tissue (steady state and time of flight)." Optical-thermal response of laser-irradiated tissue. Springer Netherlands; 109-144 (2011). 